\title{
La labor del psicólogo del deporte con la Selección Argentina de Handball Femenino en los Juegos Olímpicos de Brasill 2016
}

\author{
Laura Spaccarotella \\ Selección Argentina de Handball femenino, Argentina
}

RESUMEN: En el presente artículo desarrollaré mi primera experiencia profesional como psicóloga del deporte en unos Juegos Olímpicos (Brasil 2016), siendo también la primera participación en unos Juegos Olímpicos de la Selección Argentina de Handball Femenino. En primer lugar, se abordarála preparación psicológica previa a los Juegos, destacando la intervención con el entrenador para mejorar su desempeño con el equipo (cohesión, confianza, liderazgo y manejo de presiones del equipo) y con las jugadoras en el aprendizaje de habilidades psicológicas para la competición (diálogo interno positivo, relajación y visualización). En segundo lugar, se presenta la intervención psicológica realizada durante los Juegos Olímpicos, incidiendo en el control de la ansiedad como recurso importante, con el objetivo de mejorar el rendimiento y el bienestar de los deportistas. Y, por último, se considerarán situaciones y obstáculos que dificultan el rol del psicólogo del deporte en unos Juegos Olímpicos, con las reflexiones subsiguientes que puedan colaborar en la mejora del desempeño profesional del mismo.

PALABRAS CLAVES: Juegos Olímpicos, entrenamiento psicológico y psicólogo del deporte.

The work of the sports psychologist in the female argentine handlball team in the brazil 2016 olympic games

ABSTRACT: This article will summarize my first professional experience as a sports psychologist in an edition of the Olympic Games (Brazil 2016). This was also the first time that the female handball team of Argentina participated in an Olympiad.Psychological preparation before the Olympic Games will first first be addressed, emphasizing intervention with the coach to improve his performance with the team (cohesion, confidence, leadership, and handling team pressure), and with the players in learning of psychological skills for competition (positive internal dialogue, relaxation, and visualization).Psychological intervention during the Olympics is then discussed, focusing on control of anxiety as an important resource, in order to improve the performance and well-being of athletes.Finally, situations and obstacles that hinder the role of the sports psychologist during the Olympic Games will be discussed and will be followed by reflections that may contribute to improve the professional performance of psychologists.

KEYWORDS: Olympic games, psychological training, sports psychologist.

O traballho do psicólogo esportivo com a seleção argentina de handebol feminino nos jogos olímpicos de brasill 2016

RESUMO: Neste artigo vou expor a minha primeira experiência profissional como psicólogo do esporte en un s Jogos Olímpicos (Brasil 2016), sendo também a primeira participação numa Olimpíada da Seleção Argentina de Handebol Feminino. Primeiro, vou tratar da preparação psicológica prévia aos Jogos Olímpicos, destacando a intervenção com o treinador para melhorar o desempenho dele com a equipe (coesão, confiança, liderança e gestão de pressões da equipe) e com as jogadoras na aprendizagem da habilidade psicológica para a competição (auto-fala positiva, relaxamento e visualização). Em segundo lugar, é apresentada a intervenção psicológica levada a cabo durante os Jogos Olímpicos, frisando no controle da ansiedade como um recurso importante, a fim de melhorar o desempenho e bem-estar dos atletas. E, finalmente, serão consideradas situações e obstáculos para o papel do psicólogo do esporte nos Jogos Olímpicos, com reflexões posteriores que podem ajudar a melhorar o desempenho profissional dele.

PALAVRAS-CHAVE: Especialidade psicológica; Rol profissional; Psicologia do Esporte e do Exercício.

Artículo recibido: 28/02/2017 | Artículo aceptado: 23/05/2017

La autora es Psicóloga integrante del Cuerpo Técnico de la Selección Argentina de Handball:

La correspondencia sobre este artículo debe enviarse a la autora a c/ Blanco Encalada 3822, Ciudad de Buenos Aires (1430), Buenos Aires. Argentina. Email:

aura_spaccarorella@yahoo.con

(cc) EY-No-ND Este es un artículo Open Access bajo la licencia 
La labor del psicólogo del deporte en las olimpiadas tiene una cierta tradición (Buceta, 1992; Blanco, y Buceta, 1993; Cortés y Font, 1993; Cruz, 1992; García y Llames, 1992; Gould y Maynard, 2009; Orlick y Partington, 1987; Gonzalez-CarbaIlido, Ordoqui-Baldriche, Pineda-Alonso y Estrada-Contreras, 2013; Palmi, 1992; Pérez, Serpa y Castro, 2006), que sirve de referencia y experiencia para los diferentes profesionales del ámbito. El objetivo del presente trabajo es compartir con el lector mi desempeño y experiencias como psicóloga del deporte con la Selección Argentina de Handball Femenino en los Juegos Olímpicos de Brasil 2016, labor que llevo desarrollando con la selección desde hace más de dos años (2015/ actualidad). Esta vivencia tiene la peculiaridad que tanto para la selección como para mí fue la primera participación en este gran evento deportivo.

Al presentarse como un hito competitivo al que no siempre se accede con facilidad, y en casos favorables se clasifica cada cuatro años, es importante registrar y evaluar las experiencias, aciertos y dificultades que se manifiestan en lo que refiere a nuestro quehacer como psicólogos del deporte y las características de especificidad de la experiencia olímpica. De esta forma podremos contribuir a la mejora de la Psicología del Deporte y a la labor de los psicólogos que trabajan en este ámbito.

Es deslumbrante, excitante y maravillosa la experiencia de haber participado y vivido unos Juegos Olímpicos. Sin embargo, podemos quedarnos con esa imagen de ensueño o desandar el camino y los procesos que conducen hacia allí. Es por ello que, en primer lugar, desarrollaré las etapas previas a los Juegos Olímpicos con sus distintos momentos, temores, expectativas y la intervención psicológica realiza$\mathrm{da}$, y en segundo lugar, el trabajo durante los propios juegos, concluyendo con algunas reflexiones finales sobre mi desempeño profesional.

\section{Experiencia profesional}

\section{El trabajo psicológico previo a los Juegos Olímpicos}

Parto de la idea de que los Juegos Olímpicos comienzan mucho antes que la fecha de su celebración, porque supone un largo proceso de formación, preparación y capacitación previa en lo que respecta a incluir al entrenamiento psicológico como parte de la preparación general. Tal y como señalan Gould y Maynard (2009), el desarrollo de los atletas olímpicos, tanto deportiva como psicológicamente, requiere un proceso que puede llevar varios años. En mi caso, la preparación psicológica previa a las olimpiadas fue de un año.

Además, como indican Orlick y Partington (1987) refirién- dose a la intervención de los psicólogos del deporte con los deportistas olímpicos canadienses, es recomendable la disponibilidad del psicólogo desde tiempo antes de las competiciones más importantes, ya que, a través de las observaciones de comportamientos y situaciones efectuadas durante la convivencia y el conocimiento adquirido a través de ellas, podrá colaborar en pos de un mejor funcionamiento de los deportistas.

En una primera etapa, desde una perspectiva indirecta de intervención del psicólogo del deporte (Buceta, 1998; Blanco y Buceta, 1993), el entrenador de la selección me propuso trabajar sólo con él para mejorar el desempeño y liderazgo con las jugadoras. Una de las inquietudes era que el equipo contaba con deportistas de trayectoria profesional, pero no tenían la experiencia de las grandes citas internacionales como puede ser unos Juegos Olímpicos. Las temáticas del entrenador estaban orientadas principalmente hacia el rendimiento deportivo y se concretan en las siguientes preguntas: ¿Cómo puedo mejorar la cohesión del equipo? ¿Cómo puedo gestionar el grado de dificultad y la presión de la competición? ¿Cómo puedo fortalecer la confianza de las jugadoras?, ¿Cómo puedo potenciar mi liderazgo?, entre otras cuestiones.

Para ejemplificar esta línea de trabajo, para mejorar la cohesión del equipo, teniendo en cuenta la vertiente motivacional y social de la misma (Carron y Dennis, 2001), se trabajó en el establecimiento de objetivos individuales (jugadoras, cuerpo técnico y demás integrantes del equipo) y grupales (objetivos compartidos), así como una mejora de las relaciones interpersonales entre los miembros del equipo. En cuanto a la motivación, se realizaron encuentros grupales para conversar sobre estas cuestiones y establecer objetivos, dando respuesta a las preguntas de ¿qué quiero para mí y el equipo? y ¿cómo puedo conseguirlo?

Respecto a las relaciones, se realizó actividades para fomentar la apertura y el intercambio, el conocimiento de aspectos personales de las deportistas (intereses, valores, necesidades y motivaciones) y el establecimiento de una identidad grupal. Como ejemplo de esto último, fue tomar el nombre de "La Garra" tras una gira (partidos amistosos) que aconteció en Hungría, con la necesidad de nombrarse y ser nombradas como equipo. Se realizó una dinámica grupal en el cual se pidió a las jugadoras establecer los valores que les definía como equipo, unión, compromiso, humildad, pasión, esfuerzo y alegría fueron los que surgieron. Dicha etapa se vivió con intensa emocionalidad, con el deseo de estar en Brasil 2016.

Además de dar respuesta a estas cuestiones, es fundamental crear un vínculo y un ambiente positivo de trabajo con el entrenador, como base para el desarrollo de una labor 
profesional más productiva y satisfactoria. De una actitud favorable de parte del entrenador dependerá que nuestro trabajo como psicólogos pueda realizarse, concedernos confianza e independencia apropiadas y colaborando activamente con decisiones y acciones adecuadas, será la clave para llevar a cabo una intervención eficaz y útil (Buceta, 1998). Prueba de ello es que tras ir ganando la confianza del entrenador y el que viera los beneficios de mi intervención con él (tras 4 meses de trabajo, con una periodicidad de una sesión semanal, de una hora y media de duración aproximadamente cada una), en un segundo momento, es cuando se me propuso comenzar a trabajar también con las jugadoras y el equipo.

Cabe destacar que el equipo no tenía experiencia previa en el trabajo sistemático con una psicóloga del deporte, aunque algunas jugadoras habían recurrido a un psicólogo o terapeuta fuera del ámbito deportivo. Por esta razón, hubo un trabajo previo de información sobre el rol, funciones y objetivos de intervención del psicólogo del deporte (Cantón, 2016).

Se siguió un enfoque educativo-formativo con las jugadoras al integrar mi labor, para que éstas desarrollasen ciertas habilidades psicológicas para afrontar la competición. Desde una perspectiva positiva de la Psicología del Deporte, se busca potenciar las condiciones mentales de los deportistas y no "curar" o "tratar" problemas deportivos (Cantón, 2016; García-Naveira, 2010; Pérez et al., 1993).

En esta etapa conté con tres meses para la preparación psicológica previa a los Juegos Panamericanos de Toronto, que era el evento deportivo clasificatorio para los Juegos Olímpicos. Fue el período de trabajo más intenso y regular. Asistía dos o tres veces (según necesidades) a la semana al Centro Nacional de Alto Rendimiento Deportivo (CENARD), lugar donde entrenaba el equipo. Por un lado, realizaba sesiones individuales y grupales con las jugadoras, y por otro, continuaba manteniendo sesiones individuales con el entrenador (una vez por semana).

Fue fundamental adaptarme a las instalaciones con las que contábamos, ya que carecíamos de unas específicas para realizar mi trabajo. El lugar habitual de trabajo con el equipo fue en la cancha donde entrenan, también se utilizó la biblioteca (sala con mesas y sillas), y, ante la falta del mismo, un pasillo de las instalaciones deportivas.

El entrenador pautaba mi horario cuando tenía que trabajar con el grupo. Dentro de la planificación deportiva, se establecía antes o a término del entrenamiento, siendo esta última la menos habitual, ya que, tras el esfuerzo realizado, las deportistas quieren retirarse. Cada intervención grupal era de 40 minutos aproximadamente. Además, se realizaban citas individuales que se pactaban con las jugadoras fuera de los horarios de entrenamiento (sesiones de 40-60 minutos cada una). Estas cuestiones son importantes ya que como indican Gonzalez-Carballido, Ordoqui-Baldriche, Pineda-Alonso y Estrada-Contreras (2013), el acierto o pertinencia de las evaluaciones e intervenciones del psicólogo, dependerán de su adecuación al plan de entrenamiento deportivo y la optimización del tiempo de trabajo con los deportistas, evitando la ocupación de tiempo extra que no vaya en el sentido del necesario descanso y ocio reparador. Además, en mi opinión, tampoco tienen que ser intervenciones excesivamente largas que provoquen aburrimiento, dado que los deportistas requieren intervenciones acotadas, eficaces y dinámicas, percibidas como útiles para su desempeño.

Los aspectos a trabajar con el equipo se iban hablando y consensuando en primer lugar con el entrenador y con el resto del cuerpo técnico. Esto fue muy útil para manejar criterios comunes siguiendo una línea de trabajo coherente. Desde esta perspectiva, fue básico también el trabajo interdisciplinar para la mejora cotidiana. Como señala Palmi (1992), la visión interdisciplinar del deporte es la que nos permite intervenir con mayor eficacia dado que estamos más próximos a la demanda planteada por los diferentes técnicos con los que se relaciona el deportista.

Un aspecto básico que se trabajó con las jugadoras fue el control de la ansiedad. Se realizaron talleres en el que se les explicó los efectos negativos de la ansiedad sobre el rendimiento deportivo y se les entrenó en ciertas habilidades psicológicas para su manejo, como la relajación progresiva de Jacobson, el diálogo interno positivo y la visualización como práctica para afrontar posibles situaciones estresantes (Rosado, Dos Santos y Guillén, 2012; Weinberg y Gould, 2007).

En relación al control de la ansiedad, además, se trabajó con escenas temidas, aplicando estrategias psicodramáticas y lúdicas que apuntaban a la descripción y escenificación de situaciones conflictivas para que posteriormente posibilitara la adquisición de conductas alternativas de respuestas positivas de resolución (Pavlovsky, Kesselman y Frydlewsky, 2007). Por ejemplo, se representó el momento en que el entrenador anunciaría la lista de jugadoras que irían a un mundial. Se configuraron cuatro grupos de siete integrantes en el que tenían que representar esa situación. De esta experiencia, se hacía una puesta en común sintetizando las distintas escenas actuadas. Dicho espacio permitió crear un clima de intimidad y cohesión al lograr manifestar temores conjuntamente.

También, se intercambiaron y conversaron expectativas, miedos e inseguridades en cuanto a eventos significativos para cada una. Por ejemplo, algunas jugadoras habían decidido retirarse de la carrera deportiva después de los Juegos Olímpicos, y, mientras que, para algunas, las más jóvenes, 
podría significar un comienzo, para las mayores que se retiraban se trataba de un cierre y hasta posiblemente de un duelo psicológico. Otro tema tratado fue el temor a posibles lesiones, que era acallado, siendo éste, otro factor de estrés. El ponerlo en palabras fue una estrategia de afrontamiento efectiva.

Por otro lado, utilicé el baile como medio de expresión, con el objetivo de potenciar la creatividad, la fluidez, salirse momentáneamente de las rutinas y exigencias. El baile pone en funcionamiento músculos de diferentes partes del cuerpo que pocas veces se activan, dejando en reposo los que se usan de continuo, distrayendo, distendiendo y divirtiendo (De Prado y Charaf, 2000).

Cabe destacar que en algunas dinámicas grupales se incluyó al cuerpo técnico para fomentar la cohesión y, a la vez, para observar la asunción de roles, actitudes y la calidad de liderazgo, mientras que en la mayoría, se decidió conjuntamente con el cuerpo técnico que el "espacio" y "trabajo" sea de las jugadoras, para que haya más intimidad y expresión de las mismas.

Una vez realizado el trabajo pre-clasificatorio hacia los Juegos Olímpicos, "La Garra" fue a Toronto a los Juegos Panamericanos a buscar el pase de la clasificación. Dicho torneo duró 15 días, obteniendo la medalla de plata (segundo puesto). Tanto el equipo como el cuerpo técnico estaban unidos y compartían un clima emocional positivo. No estuve allí físicamente, aunque hubo un contacto regular con el cuerpo técnico y las jugadoras a través de distintos medios tecnológicos.

"La Garra" volvió siendo el primer equipo argentino de handball femenino en clasificar a unos Juego Olímpico. Transcurrido este primer período, la segunda etapa se inició después de un mes de vacaciones. Posteriormente se retomaron los entrenamientos, quedando 7 meses para los Juegos. Durante esta etapa se consolidó las técnicas psicológicas aprendidas y hubo numerosas charlas en relación a la especificidad de la experiencia olímpica (análisis de rivales, la convivencia en la villa olímpica, la exigencia de la competición, establecimiento de nuevos objetivos, etc.). El objetivo era llegar en un estado óptimo a la competición después del éxito de la clasificación, las vacaciones y los meses que quedaban por delante.

\section{El trabajo psicológico en los Juegos Olímpicos}

Ya en Brasil, se trató de convivir con la rutina deportiva, con la prensa, la excitación de la competición, los afectos de las personas significativas de las deportistas que viajaban y todo lo que podría actuar como distractores del objetivo deportivo. Por ejemplo, además de la carga emocional de ser sus primeros juegos olímpicos, en el caso de Argentina, dado la cercanía de nuestro país con Brasil, hizo que todas las familias, amigos, compañeros de club y parejas viajaran con el equipo. Este fue uno de los primeros objetivos a lograr, ser capaz de focalizar en la convivencia y la competición, dejando de lado todo aquello que distrajera (Hernández-Bonilla, 2016; Roffé, 2016). Se buscó dar un orden a todo esto. Los pases de visitas estaban acotados, todas querían las visitas de sus seres queridos y tuvo que compaginarse del mejor modo posible, así como también el ir a ver otras competencias.

En cuanto a la ceremonia de apertura se optó concurrir, lo cual era una decisión de cada equipo o deportista, dado que su finalización a altas horas de la noche podía afectar negativamente al rendimiento de aquellos que competían al día siguiente. Algunos autores (Gould y Maynard, 2009; Serpa y Castro, 2006) ya mencionan la ceremonia de apertura de los Juegos como una influencia medioambiental negativa en el rendimiento del deportista. En nuestro caso se decidió asistir, mientras que la Selección Argentina de Hockey Masculino, medalla de oro en las olimpíadas, había optado por no hacerlo. La Garra jugó a la noche del día siguiente, con un resultado que no le fue favorable.

La planificación del tiempo libre no es una cuestión menor. Hay que brindar libertad en un tiempo planificado. El deportista necesita "desconectar" de la convivencia reglada y la competición. Dado el corto intervalo que había entre competencia y recuperación, había que optimizar los tiempos y contar con una organización más precisa de los mismos. La villa olímpica contaba con un espacio de recreación donde se podía ver a los deportistas jugando a la play station, pool, máquinas electrónicas de juego, mullidos pufs para recostarse y descansar, etc. Algunas deportistas optaban por ir a ver competiciones, encontrase con algún familiar, descansar, etc. Fue complejo estructurar el tiempo libre por las eventualidades que se daban día a día, incluso el equipo tenía que salir de la villa para dirigirse a su lugar de entrenamiento, el cual fue cambiado en una serie de ocasiones. Como los partidos eran en franja nocturna, los entrenamientos también se realizaban durante la noche, en los días que no se jugaba.

En relación a mis intervenciones me he encontrada obstaculizada por factores de espacios y tiempos, pero traté de optimizar mi trabajo bajo las condiciones novedosas que también se presentaban a mi tarea profesional. Al igual que sucedió durante el trabajo previo a los Juegos Olímpicos, tuve que adaptarme a las instalaciones y medios con los que contaba, ya que no había despachos o salas para el trabajo del psicólogo del deporte en la villa olímpica. En variadas circunstancias de tipo informal me encontré manteniendo 
charlas con las distintas jugadoras y también con los integrantes del cuerpo técnico caminando por las instalaciones, en el área de recreación, espacios de comida, trayectos en autobús, en las habitaciones o gimnasio, aprovechando muchos momentos "vacíos".

Las intervenciones siguieron siendo por vía directa con las deportistas, como también por la vía indirecta, a través del asesoramiento al entrenador. El foco de atención estaba puesto en la convivencia, los entrenamientos y la competición.

Veinte días de concentración, era algo a considerar en lo que hacía a la convivencia, donde la aparición de algunas tensiones entre los miembros del equipo podían ser esperables. Estar atentos a potenciar las habilidades de adaptación a todo lo novedoso causado por el entorno, fomentar los niveles de tolerancia y mantener cierto grado de estabilidad nos compete como psicólogos. Esto era motivo de charlas donde se trató de evitar estas cuestiones, siempre buscando focalizar hacia los objetivos deportivos.

Previo a comenzar algunos entrenamientos se desarroIlaron dinámicas que apuntaban a crear un buen clima emocional, facilitado por los beneficios de la risa, liberadora de catecolaminas y endorfinas (Ferrer, 2013). Sustentándose en la formulación de Fredricson y Branigan (2000) según la cual las emociones positivas revierten los efectos fisiológicos de las negativas, la alegría es la emoción positiva preponderante que "La Garra" tiene como recurso para revertir situaciones de estrés.

Se trabajó en visualización para preparar los partidos, centrándose la atención en los objetivos individuales y colectivos de rendimiento de las jugadoras. También se realizó análisis post competencia de un modo constructivo, como espacio de reflexión y aprendizaje, detectando las fortalezas y las áreas del equipo. Y, por último, entre competencias se realizaban sesiones de relajación y visualización para potenciar la relajación física y las emociones positivas.

Señalar que en determinadas situaciones el equipo me solicitaba que fuera mediadora con el entrenador en relación algunos temas relevantes para ellas, por ejemplo, temas de organización de tiempos, de lugar de hospedaje o algún tema particular que yo podía adelantarle. Esta labor ha favorecido respuestas más ágiles, facilitando resolver determinadas situaciones y la labor del entrenador, opinión que él mismo trasmite al referirse a una de mis funciones.

A modo de información general, tras la valoración positiva del trabajo interdisciplinar, el proceso realizado y el esfuerzo de las propias jugadoras, los resultados deportivos no fueron positivos, no logrando pasar de ronda. No hubo un trabajo específico pos Juegos, luego de los 26 días de descanso que se dieron.

\section{Discusión}

A modo de conclusión, rescatar que el éxito olímpico es un proceso a largo plazo, complejo y frágil, que raramente sucede debido al azar y que es fácilmente perturbable debido a las múltiples distracciones existentes. Por ello se requiere de una planificación y preparación interdisciplinar para la mejora del rendimiento y el bienestar del deportista. En concreto, los Juegos Olímpicos comienzan mucho antes en la mente de las jugadoras y requiere de un aprendizaje y entrenamiento en habilidades psicológicas, para de esta forma darle continuidad en la propia competición.

Destacar la importancia del vínculo con el entrenador como base para el trabajo psicológico. En mi caso, además de la labor con el propio entrenador, esta cuestión me facilitó el acceso al equipo. Partiendo de un asesoramiento y promoción del rol del psicólogo del deporte, la mejora de la cohesión (metas y relaciones), confianza y manejo de presiones fueron cuestiones centrales. Especial atención requiere la planificación pos-clasificación a los Juegos, ya que desde ese momento hasta la propia competición habían pasado varios meses, y se requiere llegar en un estado óptimo para competir tanto a nivel técnico, táctico, físico y psicológico.

Las intervenciones (individuales y grupales) fueron adaptadas a la planificación y los tiempos deportivos (entrenamientos y competición) y personales (descanso y ocio) de los deportistas, con intervenciones acotadas, eficaces y dinámicas. A pesar de ello, se precisa una mejor organización del tiempo para el trabajo psicológico, dándole el espacio y la importancia que requiere, por ejemplo, estableciendo días y horarios fijos de trabajo, desde la flexibilidad que pueda condicionar la actividad deportiva.

También fue importante la adaptación a las instalaciones deportivas para desarrollar mi labor tanto antes como durante los Juegos, aunque en el futuro sería importante anticiparse y solicitar o crear espacios para ello (despachos y salas para las reuniones).

Dentro de la concentración en los Juegos Olímpicos es relevante planificar y gestionar la convivencia, establecer un plan conductual para evitar los distractores del evento, realizar una intervención psicológica pre y pos partido, así como el acompañamiento entre la competición. Además, hay que sacar una mayor ventaja a los tiempos libres para continuar desarrollando al equipo (p.ej., fomentando la unidad) y la atención psicológica individual.

El trabajo pos Juegos creo que es necesario, cuestión que no hemos realizado. Es importante tener espacios de reflexión y aprendizaje, rescatar lo positivo y establecer áreas de mejorar para un futuro. 
Desde una perspectiva del psicólogo del deporte como profesional, a posteriori de los Juegos he mantenido charlas con colegas que también habían participado de la experiencia olímpica con otros equipos y hemos podido compartir distintas sensaciones y experiencias como es la imprevisibilidad de ciertas situaciones que hay que resolver, el estrés que uno puede padecer por la exigencia de la competición y cierta soledad en lo que a la especificidad del rol como psicóloga se refiere.

Es por ello que, en el inter-juego constante entre la objetividad y el involucrarse con los equipos desde la convivencia que se establece, sería conveniente tener la supervisión de un colega que pueda colaborar desde una lectura desafectivizada de las cargas emocionales que se nuclean en torno a una competencia que posee características de exclusividad.

Y, por último, cómo y quiénes nos contratan, es un tema que aún no está del todo resuelto. Hay temas burocráticos relacionados a nuestra inserción y en muchos casos a una función que aún no posee la jerarquización necesaria. El desafío es continuar ocupando espacios para que la disciplina siga en vías de expansión, desarrollo y consolidación. Tenemos que educar en la práctica con los aportes que podemos brindar.

\section{Referencias}

Buceta, J. M. (1992). Intervención psicológica con el equipo nacional olímpico de baloncesto femenino. Revista de Psicología del Deporte, 2, 69-87.

Buceta, J. M. (1998). Psicología del entrenamiento deportivo. Madrid, España: Dykinson.

Blanco, I. y Buceta, J. M. (1993). Intervención psicológica con el equipo nacional olímpico de hockey hierba femenino. Revista de Psicología del Deporte, 2(1), 87-100.

Cantón, E. (2016). La especialidad profesional en Psicología del Deporte. Revista de Psicología aplicada al Deporte y al Ejercicio Físico, 1, e2. https://doi.org/10.5093/rpadef2016a2

Gonzalez-Carballido, Ordoqui-Baldriche, Pineda-Alonso y Estrada-Contreras (2013). Intervenciones psicológicas aplicadas a los deportistas olímpicos cubanos que asistieron a Londres, 2012. Cuadernos de Psicología del Deporte, 13(2), 91-100.

Carron, A.V., y Dennis, P.W. (2001). The sport team as an effective group. En J.M. Williams (ed.), Applied sport psychology: Personal growth to peak performance (pp. 120-134). Mountain View, CA: Mayfi eld.
Cruz, J. (1992). El Asesoramiento y la intervención psicológica en deportistas olímpicos. Revista de Psicología del Deporte, 2, 41-46.

Ferrer, L. (2013). Procedimiento de identificación de las emociones positivas adecuadas para revertir el estrés asociado a los tiros libres en el baloncesto. Revista Iberoamericana de Psicología del Ejercicio y el Deporte, 8(1), 16-34.

Fredrickson, B. L. y Branigan, C. (2000). Positive emotions. En T. J. Mayne y G. A. Bonanno (Eds.), Emotions: current issues and future directions (pp. 123-151). Nueva York, Estados Unidos: Guilford Press.

García, J. y Llames, R. (1992). Preparación psicológica de la selección olímpica de fútbol. Revista de Psicología del Deporte, 2, 63-66.

García-Naveira, A. (2010). El psicólogo del deporte en el alto rendimiento: aportaciones y retos futuros. Papeles del Psicologo, 31 , 259-268.

Gould, D. y Maynard, I. (2009). Psychological preparation for the Olimpic Games. Journal of Sports Sciences, 27, 1393-1408.

Hernández-Bonilla, L. (2016). La psicología del deporte en selecciones de fútbol de Costa Rica: experiencias y reflexiones. Revista de Psicología Aplicada al Deporte y al Ejercicio Físico, 1, e5. https://doi.org/10.5093/rpadef2016a5

Orlick, T. y Partington, J. (1987). The sport psychology consultant: analysis of critical components as viewed by canadian olympic athletes. The Sport Psychologist, 1(1), 4-17.

Palmi, J. (1992). Intervención psicológica para la selección nacional olímpica de hockey sobre patines. Revista de Psicología del Deporte, 2, 53-62

Pavlodsky, E., Kesselman, H y Frydlewsky, L. (2007). Las escenas temidas del coordinador de grupos. Buenos Aires: Editorial Galerna.

De Prado, D. y Charaf, M. (2000). Relajación creativa. Técnicas y claves para el entrenamiento, la competición y la práctica deportiva. Barcelona, España: Inde.

Pérez, G., Cortés, J. y Font, J. (1993). CAR: Entrenamiento psicológico para los juegos olímpicos de Barcelona 92. Revista de Psicología del Deporte, 2(1), 67-79.

Roffé, M. (2016). La preparación psicológica de la selección nacional absoluta de Colombia para el Mundial de Fútbol Brasil 2014. Revista de Psicología Aplicada al Deporte y al Ejercicio Físico, 1, e3. http://dx. doi.org/10.5093/rpadef2016a3

Rosado, A., Dos Santos, A. y Guillén, F. (2012). Estrategias de coping en jugadores de baloncesto de alta competición. Revista Iberoamericana de Psicología del Ejercicio y el Deporte, 7(1), 125-147.

Serpa, S. y Castro, T. (2006). Psicología de los Juegos Olímpicos: la percepción de los entrenadores. Revista de Psicología del Deporte, 15, 183-199.

Weinberg, R., y Gould, D. (2007). Fundamentos de psicología del deporte y del ejercicio físico. Argentina: Editorial Médica Panamericana. 